\title{
Some things are better in the upside down
}

\author{
Mark Roeser, MD, and Alexander Sasha Krupnick, MD
}

\author{
From the Lung Transplant Program and Department of Surgery, University of Virginia, Charlottesville, Va. \\ Disclosures: Authors have nothing to disclose with regard to commercial support. \\ Received for publication Sept 4, 2018; accepted for publication Sept 4, 2018; available ahead of print Oct 10, \\ 2018. \\ Address for reprints: Alexander Sasha Krupnick, MD, University of Virginia, PO Box 800679, Charlottesville, VA \\ 22908 (E-mail: sashak@virginia.edu). \\ J Thorac Cardiovasc Surg 2019;157:423-4 \\ 0022-5223/\$0.00 \\ Published by Elsevier Inc. on behalf of The American Association for Thoracic Surgery \\ https://doi.org/10.1016/j.jtcvs.2018.09.009
}

In 2001, Steen and colleagues ${ }^{1}$ described the utility of ex vivo lung perfusion (EVLP) for evaluating lung graft function after circulatory death in non-heart beating donors. In 2005 the same group ${ }^{2}$ reconditioned an initially unacceptable lung graft and transplanted it into a patient who would otherwise not have been able to benefit from transplantation. In 2011, the Toronto group ${ }^{3}$ published their landmark prospective clinical trial of 136 graft recipients, 20 of whom received lungs that had undergone EVLP, that demonstrated the safety of using EVLP to evaluate and recondition marginal grafts. This led to US Food and Drug Administration approval with the hope that the rehabilitation of marginal lungs would greatly expand the donor pool.

In the current issue of the Journal, Niikawa and colleagues ${ }^{4}$ from the Cleveland Clinic describe a simple yet elegant study with powerful conclusions in which they slightly altered the protocol for EVLP by putting an experimental porcine lung graft in a prone position during reconditioning. The prone grafts demonstrated improvement in cellular function as well as pathologic and cytokine tissue analysis. Niikawa and colleagues ${ }^{4}$ follow the Lund protocol for EVLP that uses the LS1 system (Vivoline Medical AB, Lund, Sweden), which is commonly used in Europe. American clinicians may be more familiar with the Toronto protocol that commonly uses the XPS system (XVIVO Perfusion AB, Göteborg, Sweden) or the OCS protocol that uses the OCS Lung System (TransMedics, Inc, Andover, Mass), which are more accepted in the United States. Regardless, both cohorts had lungs placed in the same system, and the prone position cohort had a better outcome.

It is fascinating that the mere positioning of the lungs can have such great consequences, but the physiology responsible for improvement in function is still unclear. Although we see this clinically when we move patients with adult respiratory distress syndrome to the prone position, the improvement in function in these patients is attributed to improved ventilation-perfusion matching. It tonight.

\section{References}



Alexander Sasha Krupnick, MD (left), and Mark Roeser, MD (right)

\section{Central Message}

Altering the position of the lung graft during ex vivo perfusion may offer an advantage for graft reconditioning.

See Article page 425 .

is also interesting that these results were obtained in a quadruped that rarely spends time with its lungs in the supine position. ${ }^{5}$ Maybe the more "physiologic" positioning of prone porcine lungs resulted in improved function? This makes one wonder whether human lungs should be perfused in an upright fashion. Another obvious question raised by this study is whether such benefits will be maintained after transplantation. Although it is fascinating that the prone lungs did better, at the end of the day, EVLP's ultimate goal is to let the physician know whether these lungs are acceptable for use in a transplant. If placement of the lungs in a prone position during EVLP yields false optimism regarding how they will perform after transplantation, will the practice lead to engraftment of suboptimal grafts? Nevertheless, if this simple technique can show a clinical benefit after transplantation, then the lung transplant community should rejoice, because this technique can easily be added to any current EVLP protocol. On the basis of these new data, and just to be on the safe side, we should all plan on sleeping in the prone position

1. Steen S, Sjöberg T, Pierre L, Liao Q, Eriksson L, Algotsson L. Transplantation of lungs from a non-heart-beating donor. Lancet. 2001;357:825-9.

2. Steen S, Ingemansson R, Eriksson L, Pierre L, Algotsson L, Wierup P, et al. First human transplantation of a nonacceptable donor lung after reconditioning ex vivo. Ann Thorac Surg. 2007;83:2191-4. 
3. Cypel M, Yeung JC, Liu M, Anraku M, Chen F, Karolak W, et al. Normothermic ex vivo lung perfusion in clinical lung transplantation. $N$ Engl J Med. 2011;364: 1431-40.

4. Niikawa H, Okamoto T, Ayyat KS, Itoda Y, Farver CF, McCurry KR. The protective effect of prone lung position on ischemia-reperfusion injury and lung function in an ex vivo porcine lung model. J Thorac Cardiovasc Surg. 2019;157:425-33.

5. Guérin C, Reignier J, Richard JC, Beuret P, Gacouin A, Boulain T, et al; PROSEVA Study Group. Prone positioning in severe acute respiratory distress syndrome. N Engl J Med. 2013;368:2159-68. 